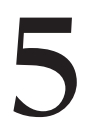

\title{
Organizational Identity and Identification During a Departmental Reorganization
}

\author{
Michael R. Mills \\ Pamela J. Bettis
}

\begin{abstract}
s noted in the previous chapter, Chapters 4 and 5 are companion pieces. This chapter shifts the focus to a second article (Mills, Bettis, Miller, \& Nolan, 2005) produced from our research project on faculty members' reactions to a reorganization of their academic department. Here, the focus is on organizational identity and organizational identification as exemplified in the new academic unit.
\end{abstract}

\section{Study Overview}

The origins of the research project and the methods used are presented in the previous chapter and are not repeated here. In the article that is the focus of this chapter (Mills et al., 2005), the findings and analyses highlight the dysfunctional interaction patterns of the new department, referred to as the Department of Educational Studies (DES), as indicated by the mode of debate, lack of trust, and fragmentation within the unit. We describe a departmental 
faculty that had substantial difficulty working together toward important collective decisions regarding matters like curricular consolidation and promotion and tenure guidelines. Themes in our analyses include truncated communications, conflict avoidance, dysfunctional decision-making processes, and our own withdrawal from participation in the unit.

Under these conditions, the department became a weak unit within which to develop an organizational identity and identification. Given the lack of mutual processing of issues, faculty members were impeded in their ability to negotiate an agreement on the identity of the unit. This inhibited sensemaking, which, in turn, hampered our identification with the new department.

\section{Theoretical Framework: Organizational Identity and Identification}

We started the study with the notion that the new unit would have to create a new organizational culture and become home to people who had not previously shared an organization. In effect, culture is the internal and symbolic context for organizational identity. Thus, the same processes of social construction and sensemaking are involved in developing and perpetuating both organizational culture and organizational identity. As such, both constructs become susceptible to reinterpretation and shifting meanings as the context for identity is reframed. Much as recent conceptions of culture have moved beyond an integrationist view to emphasize the internal variation and fragmentation (Martin, 1992), the conception of organizational identity has also moved beyond something that people take to be central, distinctive, and enduring about an organization (Albert \& Whetten, 1985) to thinking about identity as a social construction susceptible to change and incoherence (Gioia, 1998).

Organizational culture and identity, in turn, become prime considerations in organizational identification. In effect, the affiliations that we use to define ourselves are often the organizations in which we participate (Pratt, 1998). Organizational culture sets the context for organizational identity, which, in turn, says a lot about who organization members are and how they would like to think about themselves. Through organizational identification, organization members get psychological needs such as safety, affiliation, selfenhancement, and self-actualization fulfilled while the organization gets members who are more likely to act in ways congruent with organizational goals and needs. The process of identification is complicated, however, because neither the individual nor the organization has a singular identity or even consistency among multiple aspects of identities. 
Alvesson and Willmott (2002) suggest that we have not moved much beyond these recognitions to more fully grasp the identity constitution processes in organizations. They attempt to address that shortcoming in the interpretive literature, and they provide an overview of four ways individuals' identities are influenced and changed in organizations. The interactions within organizations serve to define (1) the employee by directly defining characteristics; (2) action orientations by defining appropriate work through motives, morals, and abilities; (3) social relations by defining belongingness and differentiation; and (4) fit with the social, organizational, and economic terrain.

In addition to this general framework, the paper (Mills et al., 2005) used more specific theories focused on Alvesson and Willmott's (2002) third item, explanations of identity formation via belongingness known as social identity theory (SIT) and self-categorization theory (SCT). These formulations focus on how group norms, stereotypes, and individual prototypes serve to define identities and influence identification with groups, and examine factors such as groups' homogeneity, prestige, distinctiveness, and permeability. These theories have been more formally derived and tested in broad sociological contexts, and a body of literature has grown out of attempts to extend the insights of SIT and SCT (see Hogg, Terry, \& White, 1995, for an overview of the theories and literature) to the identities of organizations (Ashforth \& Mael, 1989; Gioia, 1998) and identification within organizations (Pratt, 1998). Working from these theories, researchers hypothesize that clear and distinct differences between groups, a high salience for organizational categories in accounting for differences between groups, and membership in an attractive or prestigious group will enhance identification with a social group and organization (Hogg \& Terry, 2001; Pratt, 1998). Conversely, identification with an organization is likely to be more difficult if members are highly heterogeneous or if they are too much like members of other organizations, and the theories incorporate notions of ambivalent identification or disidentification.

Of particular interest for our study, these theories have also been used in research on mergers in organizations. Mergers are problematic because the social foundations for prior identifications usually remain intact, reducing the need for individuals to develop commitment to a new group identity. Instead, "employees of merged organizations tend to act on the basis of their premerger identity ... rather than on the basis of the identity implied by the merger" (Van Knippenberg \& Van Leeuwen, 2001, p. 251). This literature suggests that, for example, issues of in-group and out-group membership and status differentials will cause problems for organizational identification among members of the "out" or lower-status organization. Success 
in organizational mergers has been connected to conditions that promote interaction, such as equal status, intergroup cooperation, egalitarian orientation, and personal acquaintance. Given these conceptual foundations, this paper focused on the organizational factors that promote identity formation and identification within organizations and how those were manifest in a new department created by the college reorganization.

\section{Locating the Theoretical Frameworks}

The theories of organizational identity and identification have developed within the field of organizational studies, which was a major component in Michael's degree program and is the focus of his teaching in higher education administration. Much of his past research revolves around the concept of social construction of reality and sensemaking in organizations. Identity is directly involved in these processes, and the view of identity in organizational studies parallels the development of the concept of identity as a social construction in other social sciences. In this sense, the focus on organizational identity in this study does not stem from a theory in the specific sense of a formal model of relationships between variables or constructs. Rather, it is better thought of as a framework within which the topic is being discussed in the academic literature. It is this interest in questions of organizational identity and their association with organizational culture and sensemaking that preceded and motivated the entire research project.

In an edited book, Whetten and Godfrey (1998) chronicled the development of approaches to identify in the field. The chapters in that book helped shape our initial steps in applying the concepts in our study. It helped us bring together the postmodern formulation of identity and how it applies to organizational identity and the concept of identification. We continued our review of related literature as the research project progressed and, in the interest of being comprehensive and responsive to the literature, further investigated the notion of organizational identification and the more formal theoretical formulations of SIT and SCT in organizational studies. The theories specifically were the subject of a review article (Hogg et al., 1995) and an edited book (Hogg \& Terry, 2001), which included good summaries of the literature. Several directions within that literature, including the application in instances of organizational mergers and the development of a notion of "disidentification," led to optimism about the theories' applicability to our project. As discussed later in this chapter, however, that optimism later dimmed in the analysis phase of the project, when it became clear that the theories were not rich enough to explain some aspects of our findings. 
In sum, locating the framework arose from Michael's prior knowledge of the literature in the field. Further, the location of a couple of extensive literature reviews helped to summarize specific theoretical formulations and to pinpoint sets of literature for deeper review.

\section{Effects of the Theoretical Framework on the Research}

As indicated previously, the focus of the research from the beginning was on questions of identity and identification in a shifting organizational context, so we conceived of ourselves as the subjects of our research project. This made journaling appear to be the natural choice for our data gathering technique. We also wanted to avoid a situation in which the research project would significantly alter the course of the department's development. Thus, we made a deliberate attempt to keep the research project, other than the journaling, as low impact an activity as possible during the year of data collection. This meant that another data gathering technique, such as constituting ourselves as some version of a focus group at various points during the year, seemed to invite too much influence on the events we were studying. In fact, we only met as a group twice during the year, and while we did discuss substantive issues (They were hard to avoid!), the main focus was to encourage continued attention to the journaling task. We also responded to a set of common questions in our journals, as described further below.

Clearly, we had the broad framework of social construction and identity from the beginning of the study. Our specific instructions in data collection were to write about the issues the department faced and the interactions we had with faculty in formal (for example, committee meetings) and informal (hallway conversations) occasions as well as our own reactions and feelings about what we experienced.

The concern for organizational identity and identification focused journal entries on instances of interaction when members of the department were discussing issues and negotiating new understandings and expectations for their department and themselves (or finding ways not to do so). Our journaling often focused on work issues in which there were explicit discussions of the values and norms the department would embody. These instances related to both the meaning of membership in the department and how members defined their identification with DES. We also used our journals to discuss how we were feeling about the events we experienced and whether they engendered our own association with the department or people in it. Typically, the reorganization work was either tedious or acrimonious, so the journals included expressions of our disengagement with DES. 
For example, Michael—in a journal entry written December 14, 1997_ portrayed a faculty meeting in which committee members prepared briefing papers on issues to be resolved while drafting promotion and tenure (P\&T) guidelines.

The P\&T committee members kept quiet unless prompted by the faculty to discuss a topic. Even then, they seemed reluctant to speak and did not say much to help the conversation. Predictably, we spent a lot of time on the first few items and the later items got little attention at the meeting. The result is that we sometimes voted on questions that we may not have fully understood or appreciated the complexity of, and we did not always have the benefit of a full discussion to inform our vote. Thus, this exercise in pseudo-democracy really turned out to limit our ability to have full and informed input into some important decisions. The result is that I feel, and I have heard others express, that we still have not had sufficient time to discuss and deal with the issues that the P\&T document raises. This also served to limit the value of the vote and conversation for the members of the committee, since they now have to interpret written votes on paper, and they do not have the "rich media" of face-to-face conversations to inform them of how the faculty thinks about an issue.

It is easy to see how the attention to certain events and the way they are framed in the journals reflect the theoretical framework for the study, as the journal entry itself noted the missed chances at interaction that could have promoted a greater sense of shared participation in the department.

One aspect of our journaling that we adopted was that, at five points during the year, Michael asked the group members to respond to specific questions in the journals. The questions were meant to prompt some reflection on common subject matters in the journals, and the effects of the theoretical frames are evident in the questions themselves. We were asked to comment on topics such as changes we were experiencing in our professional identities, our perceived influence on our academic program and the new department, and the continuing role of our former departmental affiliations.

In the analysis phase, the specific theoretical frameworks also played explicit roles in the project direction. The analysis process began with members of the research group reading their own and another full journal and coding sections of text with a term that characterized the main idea being expressed. As a group, we then narrowed the list of codes to a set that would encompass all of the selected text sections in order to be as comprehensive and inclusive of the journal topics as possible. We then applied those codes to all the journal entries. Thus, this inductive and inclusive form of coding allowed the influence of the theoretical frame on the data collection process to roll through to the coding and sorting of the textual fragments. The codes selected 
focused on the (1) topical areas that promoted interaction in the department (promotion and tenure guidelines, selection of a chair, curricular integration), (2) modes of participation and communication among former and new colleagues, (3) roles in the new department, (4) our professional identities, (5) the view of DES from inside and outside, and (6) the status and relations of the degree programs that constituted the department. All of these issues highlight aspects of building an organizational identity for the department and our identification with it.

As the next step in the analysis process, we gathered the codes into 12 groupings based on the similarity or relatedness of the topics. Then, each set became the focus of a group meeting to interpret, derive themes and differences, and apply theoretical formulations based on the content of the text units. Michael produced a written summary of the conversations, which became the subject of further conversation, sorting and recombining the interpretations within and among the original code groupings. Eventually, the focus on interaction and sensemaking in the process of identity formation led the analysis to focus on how difficult it was to interact and create a new identity for the department. We were also led to stress missed opportunities for (and even avoidance of) communication and interaction to build a departmental identity and the retreat of faculty into the separate programs that constituted the department. Thus, in the analysis of our journals, the themes and concepts of the theoretical frameworks took on greater and more direct relevance to the data and our modes of understanding them.

\section{Critique of the Frameworks}

The events in DES reinforce the SIT/SCT position that mergers encourage the airing of differences and separation within a new unit because they heighten attention to the differences and tensions between subgroups. In addition, the general connection between the permeability of subgroups and the success of mergers is supported by negative example, since, in this unsuccessful merger, the unit quickly becomes characterized as a collection of separate programs rather than a coherent department. The role of prestige factors in this case, however, is more complicated than the SIT/SCT literature suggests. For example, the literature says that low-prestige groups would emphasize alternative characteristics related to their identity that would de-emphasize characteristics on which they were relatively weaker. In this case, research orientation and productivity-elements of academic prestige-were central issues in the conflict over promotion and tenure standards. Both high- and low-prestige programs within the department, however, opposed including 
stricter standards in the criteria language, opting instead to emphasize values of autonomy, collegiality, and egalitarianism-also highly prized among academics - in order to reinforce the independence of the various programs in the department. Thus, in this context, prestige was less of a factor in members' orientation to and identification with the new unit than these theories suggest, and egalitarianism promoted subgroup autonomy rather than group permeability.

Ultimately, the DES case urges us to think beyond the models and hypotheses of SIT/SCT approaches to organizational identity. Even while acknowledging the multiplicity and variability of identity in both people and organizations, SIT/SCT conceptions still focus identification upon the primacy of defining or prototypical characteristics and assume an integrationist view of a dominant organizational culture and identity. A broader and more comprehensive view of identity suggests, however, that there are inescapable multiplicities, complications, and even contradictions in identity. Furthermore, individuals have multiple sources of identity and different aspects of themselves that they apply with different salience in the contexts of each of their affiliations. Thus, in DES the multiple values and meanings must be combined in ways that an integrationist theory of identity would overlook.

Alvesson and Willmott's (2002) list of identification modes presented previously suggests some of the value, environmental, and contextual dimensions that are part of the identity development process and thus represents a broader viewpoint for thinking about the identity construction and identification processes in DES. This theoretical formulation led us to highlight the lack of engagement in the department and to locate the problem in the constricted communication that curtailed sensemaking and, therefore, the formation of organizational culture and identity. The framework that acknowledged the multiplicity and ambiguity of identity, without also making it an inherent problem, allowed us to look beyond differences as the source of disidentification and to suggest that the problems of interaction chronicled in our journals limited the faculty's ability to build organizational identity while still accepting differences and contradictions within the organizational culture.

\section{Possible Alternative Theoretical Frameworks}

Various frameworks for the notion of organizational culture would have clear applicability to this case, as the close association of culture and identity discussed above makes apparent. From early in the study, however, we considered the unit of analysis to be us-the five journal keepers-rather than the 
department or college as a culture. Although we did comment and interpret aspects of the units and the faculty as a whole, thus approaching a cultural analysis, the real focus was our perspectives and experiences of the new unit and their effect on our organizational identification.

We also could have used frameworks and theories from leadership and/or organizational change literatures. In fact, presentation and article reviewers have questioned why we have not made fuller use of theories from these sources. From the beginning, however, our attention was focused on the questions of faculty members' identity and how it was tied to and affected by the organizational change we were experiencing. Regarding the questions about leadership, for example, our response has been to focus on the faculty's own responsibility for developing the identity of the department and our own failures in accomplishing this, rather than on the shortcomings of the administrators involved in the organizational changes.

\section{The Use of Multiple Theoretical Frameworks}

As discussed previously, we used different theoretical frameworks in two articles from our project (see the previous chapter). Although they describe the conditions in the new department in very consistent terms, the different theoretical frameworks pushed the articles to focus on different aspects of the case and produced notable differences in the conclusions of the papers. The notion of liminality applied in the first paper (Bettis, Mills, Miller, \& Nolan, 2005 ) directed the focus to the social, political, and economic contexts of the departmental change and to an externally derived definition of faculty work. The focus on organizational identity and identification in the second paper produced an emphasis on the internal construction of values, expectations, and belonging that resulted in greater attention to the formation of internal agreements such as the debate over the department's promotion and tenure policies.

Weick (1995) and Chapter 4 make the point that there are really two different types of problems during sensemaking in organizations-one when there are not enough interpretations for members to work with and the other when there are too many interpretations floating around. In our department, we experienced both problems, and the two different theoretical frameworks each highlight one of the problem types. The article on liminality (Bettis et al., 2005) showed that the faculty was constrained, in part, by the fact that there were only two characterizations of the faculty job that we were stuck between - the modernist searcher for Truth and the postmodern laborer in the knowledge factory. The article concludes by discussing the need to 
develop more interpretations of what the faculty job can be, something we were not successful at accomplishing during the reorganization. The paper on organizational identity and identification (Mills et al., 2005) showed that the new department's faculty had many different perceptions and values to bring to discussions about promotion and tenure, curriculum, and other internal matters. That article concluded that we needed to have better conditions for communication in order to work together to negotiate and better reconcile the different approaches to and interpretations of our organization and its purposes.

Thus, the use of different frameworks allowed us to have greater breadth in our analyses of the situation we studied. In effect, we were able to consider several of Alvesson and Willmott's (2002) different ways identities are influenced in organizations: (1) the fit with social and organizational contexts through the liminality framework, and (2) orientation through values and belongingness through organizational identity and identification. In addition, the two versions exposed how the lack of imagined alternatives in one realm limited choices for identity, whereas the wealth of alternatives and constricted interactions in another realm constrained sensemaking and identity construction.

\section{Additional Comments}

In addition to the pervasive influence theoretical frameworks have on the conduct and focus of the research, we also want to emphasize ways in which they contribute to one type of problem as well as provide a solution to another problem for qualitative research. On the one hand, the impact of theoretical frameworks on every phase of qualitative research contributes to one of the stickiest problems for the trustworthiness of qualitative research. On the other hand, theoretical frameworks provide the pathway to overcome the local, contextualized nature of data collection and to allow a form of generalization that some say qualitative research lacks.

If we are as beholden to our theoretical frameworks as the premise of this book and this chapter suggests, then we also have to be concerned that these frameworks become self-fulfilling prophecies. There is a real danger that they guide our data collection by determining where we direct our attention as well as the very terms we use to characterize what we see, and they turn the analyses toward conclusions that could be theorized before the research began. In qualitative research, our mechanisms for analytical skepticism are not as strong or explicit as those developed for quantitative research (and even in quantitative research, the mechanisms are limited and also serve to 
hide certain preconceptions). This places an extra obligation on qualitative researchers to question ourselves; to seek out alternative data, interpretations, and explanations; and to doubt our framings and understandings. We need to search out data that may contradict our expectations and promote change in our own understandings. Obviously, this does not mean that the theoretical framework stops directing the study. On the contrary, it suggests that the framework directs the study in two directions-both toward and away from the perceptions and concepts of the framework. Only when we do that can we push our analyses to more nuanced stages that go beyond what our frameworks provide us. And when qualitative researchers have accomplished this, we can be assured of the restored trustworthiness of our data and analyses.

Theoretical frameworks also contain the breadth that our data do not have. Theories allow our intense, specific data collection and contextualized interpretations and conclusions to speak to broader issues and conceptual formulations. As Wexler (1992) noted,

The theory is really a 'fusion of horizons' between the elements of the analytic fields as I blended them and what the subjects of the study said and did. I let the subjects lead me to the language and understandings that could only be found on the field of perception for which I had prepared. (p. 6)

By working back and forth between the detailed data and the broader concepts of the framework, researchers and readers can highlight why research can be relevant for other contexts and social settings. Qualitative researchers often talk about the continual comparisons and multiple iterations they perform with their data during analysis, but the theoretical framework is also part of this iterative process-the part that adds greater weight to the mundane data we gather and pore over.

Another way to think about this expansive process is to consider the researcher at the conjunction of two different communities of discourse. In effect, the researcher is involved in social constructions via interactions with the participants in the study and with intellectual colleagues that have helped formulate the framework being used in the study. The study involves both making sense of the research site and the participants' meanings in the context of larger theoretical ideas as well as making sense of the larger theoretical framework in light of the specific things learned in the research. Each perspective informs the other as the researcher brings both to bear in the interpretive process, and the multiple layers of the conversation make the research context speak to issues and in terms meaningful beyond its boundaries. 


\section{References}

Albert, S., \& Whetten, D. (1985). Organizational identity. In L. L. Cummings \& B. Staw (Eds.), Research in organizational behavior (Vol. 7, pp. 263-295). Greenwich, CT: JAI.

Alvesson, M., \& Willmott, H. (2002). Identity regulation as organizational control: Producing the appropriate individual. Journal of Management Studies, 39, 619-644.

Ashforth, B. E., \& Mael, F. A. (1989). Social identity theory and the organization. Academy of Management Review, 14(1), 20-39.

Bettis, P. J., Mills, M., Miller, J., \& Nolan, R. (2005). Faculty in a liminal landscape: A case study of a college reorganization. Journal of Leadership and Organizational Studies, 11(3), 47-61.

Gioia, D. (1998). From individual to organizational identity. In D. Whetten \& P. Godfrey (Eds.), Identity in organizations: Building theory through conversations (pp. 17-31). Thousand Oaks, CA: Sage.

Hogg, M., \& Terry, D. (2001). Social identity theory and organizational processes. In M. Hogg \& D. Terry (Eds.), Social identity processes in organizational contexts (pp. 1-12). Philadelphia: Psychology Press.

Hogg, M., Terry, D., \& White, K. (1995). A tale of two theories: A critical comparison of identity theory with social identity theory. Social Psychology Quarterly, 17(1), 255-269.

Martin, J. (1992). Cultures in organizations: Three perspectives. New York: Oxford University Press.

Mills, M., Bettis, P. J., Miller, J., \& Nolan, R. (2005). Experiences of academic unit reorganization: Organizational identity and identification in organizational change. Review of Higher Education, 28(4), 597-619.

Pratt, M. (1998). To be or not to be? Central questions in organizational identification. In D. Whetten \& P. Godfrey (Eds.), Identity in organizations: Building theory through conversations (pp. 17-31). Thousand Oaks, CA: Sage.

Van Knippenberg, D., \& Van Leeuwen, E. (2001). Organizational identity after a merger: Sense of continuity as the key to postmerger identification. In M. Hogg \& D. Terry (Eds.), Social identity processes in organizational contexts (pp. 249-264). Philadelphia: Psychology Press.

Weick, K. E. (1995). Sensemaking in organizations. Thousand Oaks, CA: Sage.

Wexler, P. (1992). Becoming somebody: Toward a social psychology of school. Washington, DC: Falmer.

Whetten, D., \& Godfrey, P. (1998). Identity in organizations: Building theory through conversations. Thousand Oaks, CA: Sage. 